\title{
Efficacy and safety of manual acupuncture manipulations with different frequencies on epigastric pain syndrome (EPS) in functional dyspepsia (FD) patients: study protocol for a randomized controlled trial
}

Shou-hai Hong ${ }^{1 *+}$, Sha-sha Ding ${ }^{2+}$, Fei Wu ${ }^{3}$, Ying Bi ${ }^{1}, \mathrm{Fu} \mathrm{Xu}^{1}$, Yi-jia Wan ${ }^{1}$ and Li-hua Xuan ${ }^{1 *}$

\begin{abstract}
Background: Manual acupuncture (MA) manipulations are one of the key factors influencing acupuncture effects in traditional Chinese medicine theory. Different MA manipulations contain different stimulating parameters, thus generating different acupuncture responses or effects. Evidence has demonstrated that acupuncture is effective for functional dyspepsia (FD). However, the effects of different stimulating parameters of MA manipulations on FD remain unclear.

Methods/design: This study is a randomized controlled trial with a four-arm, parallel-group structure. Patients with FD with epigastric pain syndrome (EPS) will be included and randomly allocated into four groups: three MA manipulation groups (separately treated with a frequency of $1 \mathrm{~Hz}, 2 \mathrm{~Hz}$, or $3 \mathrm{~Hz}$ ) and a control group. All groups will receive omeprazole as a basic treatment and acupuncture: in the MA manipulation groups, the needles will be manipulated manually with three different frequencies on the basis when de qi is reached, while in the control group, the needles will be inserted without any manipulation. All patients will receive acupuncture treatment of five consecutive sessions per week for 2 weeks and be followed up at 4, 8, and 12 weeks. The primary outcomes of the study include patients' response to the treatment. The secondary outcomes include dyspeptic symptoms, quality of life, mental status, fasting serum gastrin, motilin, and ghrelin concentrations, and adverse events. The protocol was approved by the Ethics committee of the First Affiliated Hospital of Zhejiang Chinese Medical University (2016-K-057-01).
\end{abstract}

Discussion: The aim of this study is to evaluate the efficacy and safety of MA manipulations with different stimulating parameters (different frequencies) on EPS in patients with FD.

Trial registration: Chinese Clinical Trial Registry, ChiCTR-IOR-16008189. Registered on 30 March 2016.

Keywords: Functional dyspepsia, Epigastric pain syndrome, Manual acupuncture, Lifting-inserting, Manipulations

\footnotetext{
*Correspondence: hongshouhai@163.com; xlh1083@163.com

${ }^{\dagger}$ Equal contributors

${ }^{1}$ Acupuncture Department, Zhejiang Provincial Hospital of TCM, Hangzhou,

China

Full list of author information is available at the end of the article
} 


\section{Background}

\section{Introduction}

Functional dyspepsia (FD) is a common disease causing upper abdominal symptoms occurring in the absence of organic disease that is likely to explain them. According to the Rome III criteria, FD is divided into two subgroups: epigastric pain syndrome (EPS) and postprandial distress syndrome (PDS) [1]. FD has negative impacts on health and also results in a high economic burden [2]. Standard management of FD has not yet been established, and satisfactory medications are also unavailable [3]. The management of FD remains a major issue in health clinics.

In recent years, evidence-based medicine has demonstrated that acupuncture is beneficial for relieving dyspepsia symptoms and associated negative emotions, thus improving patient quality of life [4-6]. The acupoints and acupuncture stimulus such as manual acupuncture (MA) manipulations are the key elements inducing the effect. Related research studies on treating FD with acupuncture usually focus on the specificity or compatibility of acupoints [6, 7]. However, the MA manipulations to treat FD have not been fully investigated, causing difficulties in the establishment and application of the best acupuncture treatment for FD. Researchers had showed that different MA manipulations contain different stimulating parameters, thus generating different acupuncture responses or effects [8-10]. Our previous study also demonstrated that lifting-inserting MA manipulations with a frequency of 2 or $3 \mathrm{~Hz}$ could better inhibit acute visceral nociception in rats with gastric distension than manipulations with a frequency of 0.5 or $1 \mathrm{~Hz}$ [11]. Therefore, we assume that the effects of MA manipulations with different stimulating parameters on EPS in FD are different. The aim of this study is to evaluate the different efficacies and safety of lifting-inserting MA manipulations with different frequencies on EPS in patients with FD.

\section{Hypothesis}

Based on our previous study, our hypothesis is that MA manipulations with different frequencies have different effects on dyspepsia symptoms and quality of life, and that the high frequency will have a better effect than the low frequency.

\section{Objectives}

The trial objectives are to clarify the different therapeutic effects and possible biological mechanisms of lifting-inserting MA manipulations with different frequencies on EPS in patients with FD.

\section{Methods/design \\ Design}

A four-armed parallel randomized controlled trial will be conducted at the Department of Acupuncture and Moxibustion, Zhejiang Provincial Hospital of TCM. The flow diagram of the entire trial is shown in Fig. 1. The Standard Protocol Items: Recommendations for Interventional Trials (SPIRIT) 2013 checklist is given in Additional file 1.

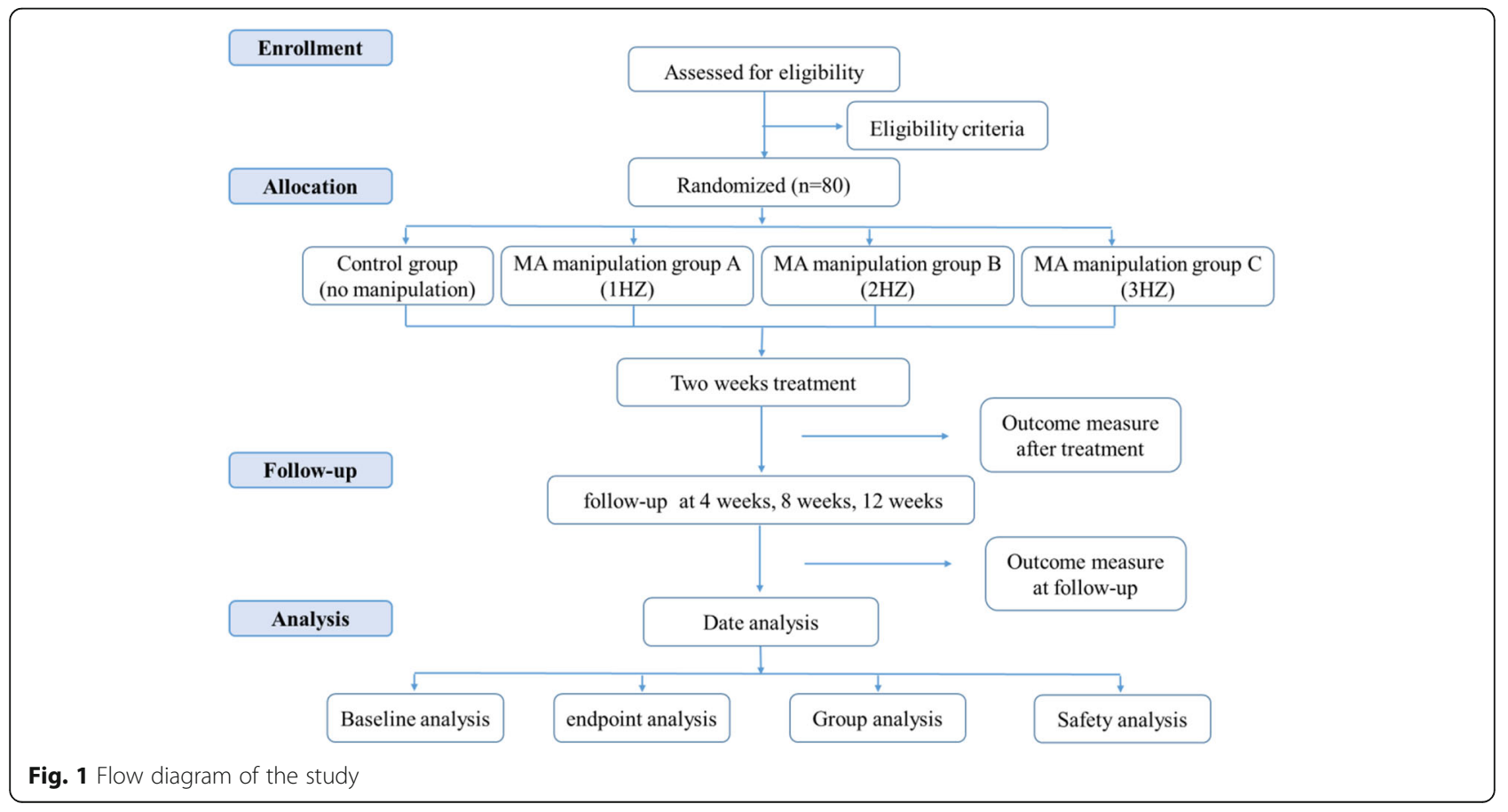




\section{Participants}

\section{Inclusion criteria}

Participants will be included if they: (1) meet the Rome III FD criteria [1] (the new diagnostic standard for FD) and have clinical symptoms of epigastric pain syndrome (EPS); (2) are between the ages of 18 and 60 years; (3) have received no other treatments related to the gastrointestinal system 1 week before enrollment and during the study; (4) are willing to agree with the study protocol and sign a written informed consent.

\section{Exclusion criteria}

Participants who present any of the following criteria will be excluded: (1) serious structural disease (disease of heart, lung, liver, kidney, digestive system, or hematopoietic system) or mental illness; (2) other diseases that could interfere with acupuncture treatment, e.g., clotting disorders or leukopenia, active skin infection, pacemaker, epilepsy, or anticoagulant therapy; (3) women who are pregnant or breastfeeding; (4) difficulties in attending the trial (e.g., paralysis, cancer, dementia, drug addiction, time constraints, surgical operation); (5) difficulty being followed up.

\section{Recruitment, randomization, allocation concealment, and blinding}

The participants will be recruited through advertisements in hospital websites and bulletin boards. The participants who meet the inclusion criteria and have signed written informed consents will be randomly assigned to one of three MA manipulation groups (groups $\mathrm{A}-\mathrm{C}$ ) or the control group in a 1:1:1:1 ratio by central randomization performed by an independent statistician from the Tigermed Pharmaceutical Science and Technology Co., Ltd. Random numbers will be generated using the PROC PLAN of SAS 9.2 (SAS Institute Inc., Cary, NC, USA). Opaque sealed envelopes will be used to conceal the group to which participants have been allocated. Investigators who have contact with the participants should be unaware of the random allocation. To preserve masking, only the acupuncturists will have access to the treatment allocation. The participants, recruiters, and outcome assessors (the evaluator and the statistician) will be unaware of the group assignments.

\section{Intervention}

Participants will be randomly allocated into one of four groups: MA manipulation group $\mathrm{A}, \mathrm{B}, \mathrm{C}$ or the control group. Everyone in each group will receive basic treatment for FD (omeprazole, a classic acid suppressing drug, is effective for the treatment of FD; $20 \mathrm{mg}$ of omeprazole $2 \times$ daily, $30 \mathrm{~min}$ before meals) and acupuncture after randomization. The only difference in the four groups is the acupuncture manipulation (e different stimulating parameters of the MA manipulations).

\section{Control group}

The needles will be inserted into the same acupoints and depth as for the MA manipulation groups but without any manipulation. The treatment period or follow-up is also the same as for the MA manipulation groups.

\section{MA manipulation groups $A, B$, and $C$}

The main acupoints ST36, PC6, and RN12 will be needled in each group. Additional acupoints will also be used based on symptom differentiation. For stagnation of liver qi, RN17 and LR13 (bilateral) are added; for deficiency of spleen qi and stomach qi, one adds BL20 (bilateral) and BL21 (bilateral); for stomach disorder due to liver qi, LR14 (bilateral) and LR3 (bilateral) are added; for damp heat in middle Jiao, one adds SP9 (bilateral) and ST44 (bilateral). Acupoints will be localized according to the 2008 World Health Organization standards [12]. Sterilized stainless steel needles $(\Phi 0.25 \mathrm{~mm} \times 25$ $\mathrm{mm}$ or $0.25 \mathrm{~mm} \times 40 \mathrm{~mm}$, Tianjin Hua Hong Medical Co., Ltd., Tianjin, China) will be used for all acupuncture procedures. The length of the needle will be chosen according to the acupoint.

After a de qi sensation is achieved, the needles in MA manipulation groups $\mathrm{A}, \mathrm{B}$, and $\mathrm{C}$ will be respectively manipulated manually with three different frequencies $(1,2$, or $3 \mathrm{~Hz})$ of lifting-inserting MA manipulations (see Table 1). The lifting-inserting MA manipulation procedure is shown in Fig. 2. Briefly, after de qi, the liftinginserting MA manipulation will be performed for 1 minute, three times, separated by 10 -minute intervals. The entire procedure takes 33 minutes. The acupuncture treatment consists of 10 sessions over a period of 2 weeks (one session per day, five continual sessions per week, with a 2-day interval between the 2 weeks) after randomization. All participants will be followed up three times in 10 weeks after treatment (at 4, 8, and 12 weeks).

All acupuncture procedures will be performed by the same licensed acupuncturist who has more than 5 years

Table 1 Parameters of three different lifting-inserting MA manipulations in MA manipulation groups

\begin{tabular}{lllll}
\hline $\begin{array}{l}\text { MA } \\
\text { manipulation } \\
\text { group }\end{array}$ & $\begin{array}{l}\text { Frequency } \\
(\mathrm{Hz})\end{array}$ & Operation of MA & $\begin{array}{l}\text { Depth } \\
(\mathrm{mm})\end{array}$ & Duration (s) \\
\hline Group A & 1 & $\begin{array}{l}\text { Neutral reinforcement } \\
\text { and reduction }\end{array}$ & $2-3$ & 60 \\
Group B & 2 & & \\
Group C & 3 & & \\
\hline
\end{tabular}

MA Manual acupuncture

In neutral reinforcement and reduction, the needle is perpendicular to the skin, and the force and amplitude of the lifting-inserting displacement is uniform; the lifting-inserting distance is $2-3 \mathrm{~mm}$ 
MA: Manual acupuncture

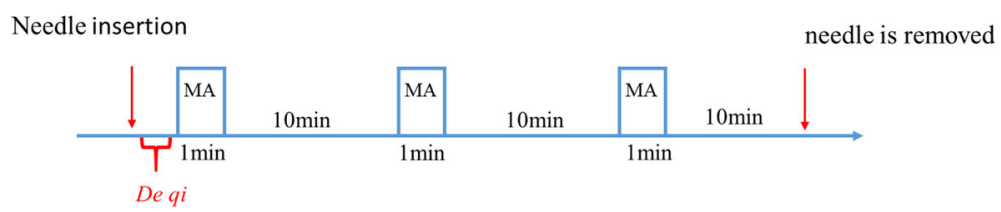

Fig. 2 The lifting-inserting MA manipulation procedure

of clinical experience. The acupuncturist will use a metronome to maintain the rhythm. Before applying the needling manipulations, the acupuncturist will repeatedly practice the manipulations on an ATP-II acupuncture manipulation parameter tester (manufactured by Shanghai University of Traditional Chinese Medicine, Shang Xin Medical Technology Company). The ATP-II device is used to measure various manual acupuncture manipulation parameters through electrical resistancesensing technology. MA manipulation waveforms appear in real time on the computer screen. During the practice phase, the acupuncturist regulates his manipulations according to the waveforms shown on the computer screen until the waveforms became reproducible. This practice ensures that the angle, depth, and frequency of lifting-inserting manipulation are consistent and repeatable.

\section{Outcomes}

The primary outcomes of the study include patients' response to the treatment [13]. The secondary outcomes include dyspeptic symptoms [14], quality of life [15], mental status [16, 17], fasting serum gastrin, motilin, and ghrelin concentrations, and adverse events. Each outcome variable will be assessed before and after treatment, and follow-up will be conducted at 4, 8, and 12 weeks in all groups (Fig. 3).

\section{Primary outcomes}

Patients' response to the treatment will be measured by an improved response rate (IRR) [13]: for EPS, the improvement of at least two scores and/or no occurrence of epigastric pain and epigastric burning included in the scale are regarded as positive responses. The Symptom Index for EPS is a 4-indicator scale assessing two main dyspepsia symptoms (epigastric pain and epigastric burning) with a scale ranging from 0 to 3 . A score of 0 indicates symptoms not present; 1 indicates occasional and mild symptoms that do not interfere with social activities; 2 indicates prolonged and obvious symptoms interfering with work or rest; and 3 indicates severe symptoms with an impact on work or rest. The IRR is the percentage of patients who are symptom-free or have shown improvement in at least two scores at the end of the treatment and follow-up.

\section{Secondary outcomes}

Dyspeptic symptoms will be assessed by the Chinese version of the Nepean Dyspepsia Index (NDI) [14], including the intensity, frequency, and level of interference of postprandial fullness, early satiety, epigastric pain, and epigastric burning sensation. The intensity of each symptom is graded and scored as follows: 0, absent; 1 , mild; 2 , moderate; 3 , severe; 4 , critical. The frequency of each symptom is also graded: 0 , absent; 1 , occasionally (1-2 days/week); 2, sometimes (3-5 days/week); 3, frequently (every day, but intermittent symptoms); 4 , continuous symptoms. The level of interference of each symptom is scored and graded as follows: 0, none; 1 , mild interference; 2, moderate interference; 3 , severe interference; 4 , critical interference. The number in front of each grading indicates the score of the corresponding symptom; the score for each symptom in the checklist of cardinal dyspeptic symptoms is calculated by adding its scores in the corresponding frequency, severity, and level of discomfort. The dyspeptic symptom sum score (DSSS) is the sum score of the four symptoms in the checklist.

The quality of life will be evaluated by the Short-Form 36 questionnaire (SF-36) [15]. Mental status will be measured by the Zung Self-Rating Depression Scale (SDS) [16] and Self-Rating Anxiety Scale (SAS) [17]. The SF-36 measures quality of life (QoL) across eight domains; the score of each domain $=[$ (actual raw score - lowest possible raw score)/raw score range] $\times 100$. For the SDS score, the following equation is used: SDS Index $=$ Raw Score $\times 1.25$. The grading of the SDS is as follows: SDS Index less than 53 points is considered normal, 53-62 is considered mild depression, 63-72 as moderate depression, and 73 and higher as severe depression. For SAS grading, the following categories are used: normal range (less than 50), mild anxiety (50 to 59), moderate anxiety (60 to 69), and severe anxiety (70 and higher).

Fasting serum levels of gastrin, motilin, and ghrelin will be measured by the enzyme-linked immunosorbent assay. A fasting venous blood sample will be drawn from the basilic vein prior to breakfast early in the morning before and after treatment. 


\begin{tabular}{|c|c|c|c|c|c|c|c|}
\hline \multirow[b]{3}{*}{ TIMEPOINT (week) } & \multicolumn{7}{|c|}{ STUDY PERIOD } \\
\hline & \multirow{2}{*}{$\begin{array}{c}\text { Enrolment } \\
-1\end{array}$} & \multirow{2}{*}{$\begin{array}{c}\text { Allocation } \\
\mathbf{0}\end{array}$} & \multicolumn{4}{|c|}{ Post-allocation } & \multirow{2}{*}{$\begin{array}{c}\text { Close-out } \\
12\end{array}$} \\
\hline & & & 1 & 2 & 4 & 8 & \\
\hline \multicolumn{8}{|l|}{ ENROLMENT: } \\
\hline \multirow{3}{*}{$\begin{array}{l}\text { Eligibility screen } \\
\text { Informed consent }\end{array}$} & $x$ & & & & & & \\
\hline & $x$ & & & & & & \\
\hline & & $x$ & & & & & \\
\hline \multicolumn{8}{|l|}{ INTERVENTIONS: } \\
\hline \multirow{4}{*}{$\begin{array}{l}\text { (MA manipulation groups } \mathrm{A} \text { ) } \\
\text { (MA manipulation groups } \mathrm{B} \text { ) } \\
\text { (MA manipulation groups } \mathrm{C} \text { ) } \\
\text { (control group) }\end{array}$} & & & - & $\rightarrow$ & & & \\
\hline & & & 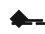 & $\Rightarrow$ & & & \\
\hline & & & 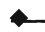 & $\rightarrow$ & & & \\
\hline & & & $\curvearrowright$ & $\rightarrow$ & & & \\
\hline \multicolumn{8}{|l|}{ ASSESSMENTS: } \\
\hline \multirow{2}{*}{$\begin{array}{l}\text { (Sociodemographic data) } \\
\text { (Improved response rate) }\end{array}$} & $x$ & $x$ & & & & & \\
\hline & & $x$ & & $x$ & $x$ & $x$ & $x$ \\
\hline \multirow[t]{2}{*}{ (Nepean Dyspepsia Index ) } & & $x$ & & $x$ & $x$ & $x$ & $x$ \\
\hline & & $x$ & & $x$ & $x$ & $x$ & $x$ \\
\hline \multirow{2}{*}{$\begin{array}{l}\text { (Zung Self-Rating Depression Scale) } \\
\text { (Self-Rating Anxiety Scale) }\end{array}$} & & $\times$ & & $x$ & $x$ & $x$ & $\times$ \\
\hline & & $x$ & & $x$ & $x$ & $x$ & $x$ \\
\hline \multirow[t]{2}{*}{ (gastrin, motilin and ghrelin) } & & $\times$ & & $x$ & & & \\
\hline & & & $x$ & $x$ & $x$ & $x$ & $x$ \\
\hline
\end{tabular}

Fig. 3 Schedule of enrollment, interventions, and assessments

Any adverse reactions of patients will be recorded in the case report form (CRF), including swelling, pain, bruise at the sites of needle insertion, or discomfort, palpitation, dizziness, etc., after acupuncture.

\section{Sample size}

The sample size calculation is based on the results of trials by Li et al. [18] and Ji et al. [19] and the recommendation of acupuncture specialists in China. The mean value of the NDI score in the $2.5 \mathrm{~Hz}$ group is 82.28 , the standard deviation is 6.97 ; the mean value of the NDI score in the $2 \mathrm{~Hz}$ group is 86.73 , the standard deviation is 7.10; the mean value of the NDI score in the $1 \mathrm{~Hz}$ group is 76.50 , the standard deviation is 8.06 ; the mean value of the NDI score in the control group is 65.85 , the standard deviation is 16.47 . To detect a significant difference between any two groups with a power of $90 \%$ and a type I error of $5 \%$, the calculated number of patients is 17 . Considering a $30 \%$ dropout rate, the total sample size required is 88 patients.

\section{Statistical analysis}

The statistical analysis will be performed by two independent statisticians. The statisticians are blinded to treatments and study protocol. Statistical analysis will be conducted on the basis of an intention-to-treat (ITT) analysis. Missing values will be imputed by the last observation carried forward (LOCF) method. Categorical data will be analyzed with the McNemar chi-square test.
Continuous data will be analyzed by analysis of variance (ANOVA). If the data trend over time and over time by treatment interactions, the repeated-measures ANOVA will be used. A $P$ value less than 0.05 is regarded as statistically significant.

\section{Quality control and dropout criteria}

All researchers will be required to undergo special training, including trial design, patient inclusion and exclusion criteria, and proper completion of the CRF. All practitioners, who have majored in acupuncture and received an acupuncture degree, are qualified doctors of traditional Chinese medicine. Patients who want to stop participating in this study, do not complete the treatment over two visits, violate the study protocol, withdraw consent for participation, or use prohibited treatments for FD will be dropped from the study. The reasons for dropouts and withdrawals from the study will be fully recorded.

\section{Discussion}

This is a protocol for a randomized, double-blind, controlled trial on MA manipulations with different stimulating parameters on EPS in patients with FD. According to the Rome III criteria, FD can be divided into two subgroups: epigastric pain syndrome (EPS) and postprandial distress syndrome (PDS) [1]. The differences in pathological mechanisms between EPS and PDS have been discussed controversially in the past. However, some new research studies have shown that these two 
subgroups differ in risk factors and pathophysiology, so their pathological mechanisms are different [20,21]. EPS and PDS should be treated with different approaches [22]. The diagnosis and treatment guidelines of dyspepsia in China also point out that clinical trials should separately study EPS and PDS. At present, acupuncture is widely used to treat disease pain, and acupuncture analgesia has gained worldwide acceptance. Therefore, we have chosen FD patients with EPS for this research.

Creating proper control groups in acupuncture clinical research is a conundrum. Rather than look for a better placebo, we designed the trial so that all patients in each group would receive acupuncture using the same needles, inserted to the same depth at the same acupoints. What differed between the groups is the frequency to which the needles are manipulated. In the control group, the needles will be inserted without any manipulation. The comparison is between four techniques. Because each patient will receive some kind of acupuncture, it is less likely that expectations will form a large part of the therapeutic effect.

Acupuncture has been accepted to effectively treat diseases by the insertion of needles into specific acupoints, and the effect is manifested when the inserted needles are manipulated by hand (manual acupuncture, MA) [23-25]. MA, consisting of needle manipulations by hand, such as lifting, thrusting, twisting, twirling, or other complex combinations, is more traditional and widely used in practice. Lifting-thrusting and twisting-twirling are the most basic needle manipulations in MA. Researchers found that different MA manipulations, containing different stimulation parameters (including frequency, angle, and depth, etc.) [8], could produce different physiological responses $[9,10]$ and therapeutic effects [26, 27]. Lifting-thrusting needle manipulation was more suitable for the treatment of gastrointestinal movement disorders than twistingtwirling manipulation [28, 29]. In our previous study, we demonstrated that lifting-inserting needle manipulations with a frequency of 2 or $3 \mathrm{~Hz}$ could better inhibit acute visceral nociception in rats with gastric distension than manipulations with a frequency of 0.5 or $1 \mathrm{~Hz}$ [11]. Therefore, we assume that the effects of lifting-inserting manipulations with different stimulation parameters (different frequencies) on EPS in FD were distinct. In order to illuminate the rule and mechanism of lifting-inserting manipulations with different frequencies treating FD with EPS, we designed the randomized controlled double-blind trial to reveal the difference in the effects of lifting-inserting manipulations with different frequencies. At the same time, we will observe some related hormone concentrations and analyze the relations in effect, hormones, and different manipulations. Thus, we can better optimize and standardize the acupuncture protocols to guide the treatment of FD with EPS.
Trial status

Participant recruitment is currently ongoing.

\section{Additional file}

Additional file 1: SPIRIT checklist. (DOCX $24 \mathrm{~kb}$ )

\begin{abstract}
Abbreviations
ANOVA: Analysis of variance; CRF: Case report form; DSSS: Dyspeptic symptom sum score; EPS: Epigastric pain syndrome; FD: Functional dyspepsia; IRR: Improved response rate; ITT: Intention to treat; LOCF: Last observation carried forward; MA: Manual acupuncture; NDI: Nepean Dyspepsia Index; QoL: Quality of life; SAS: Self-Rating Anxiety Scale; SDS: SelfRating Depression Scale; TCM: traditional Chinese medicine
\end{abstract}

\section{Acknowledgements}

Not applicable.

\section{Funding}

This study was supported by the Traditional Chinese medicine science and technology project of Zhejiang Province (grant number 2016ZA810), the National Public Special Fund for Traditional Chinese Medicine-scientific Research (grant number 201507006-01), and the National Natural Science Foundation of China (grant number 81603677).

\section{Availability of data and materials}

Not applicable.

\section{Authors' contributions}

SHH, SSD, FW, YB, XF, YJW, and LHX all contributed to the development of the study protocol. SHH and SSD drafted the manuscript. All authors read and approved the final manuscript before submission.

\section{Competing interests}

The authors declare that they have no competing interests.

Consent for publication

Not applicable.

\section{Ethics approval and consent to participate}

The study protocol was approved by the Ethics committee of the First Affiliated Hospital of Zhejiang Chinese Medical University (2016-K-057-01) and registered in the Chinese Clinical Trial Registry before the first participant was included (registration number: ChiCTR-IOR-16008189). Written informed consent will be obtained from each participant. Participants will be able to quit at any time during the study period.

\section{Author details}

${ }^{1}$ Acupuncture Department, Zhejiang Provincial Hospital of TCM, Hangzhou, China. ${ }^{2}$ Rehabilitation Department, Tianjin Nankai Hospital, Tianjin, China.

${ }^{3}$ Traditional Chinese Medicine Department, The First Hospital of Wuhu city, Wuhu, Anhui, China.

Received: 16 November 2016 Accepted: 16 February 2017 Published online: 06 March 2017

\section{References}

1. Drossman DA, Rome DDL, III. New standard for functional gastrointestinal disorders. J Gastrointestin Liver Dis. 2006;15:237-41.

2. Choi MG, Rhee PL, Park H, Lee OY, Lee KJ, et al. Randomized, controlled, multi-center trial: comparing the safety and efficacy of DA9701 and itopride hydrochloride in patients with functional dyspepsia. J Neurogastroenterol Motil. 2015;21:414-22.

3. Jung HK, Lee KJ, Choi MG, Park H, Lee JS, et al. Efficacy of DA-9701 (Motilitone) in functional dyspepsia compared to pantoprazole: a multicenter, randomized, double-blind, non-inferiority study. J Neurogastroenterol Motil. 2016;22:254-63. 
4. Zhou W, Su J, Zhang H. Efficacy and safety of acupuncture for the treatment of functional dyspepsia: meta-analysis. J Altern Complement Med. 2016;22:380-9.

5. Kim KN, Chung SY, Cho SH. Efficacy of acupuncture treatment for functiona dyspepsia: a systematic review and meta-analysis. Complement Ther Med. 2015:23:759-66

6. Jin $Y L$, Zhao Q, Zhou KH, Jing XH, Yu XC, et al. Acupuncture for functional dyspepsia: a single blinded, randomized, controlled trial. Evid Based Complement Alternat Med. 2015. doi:10.1155/2015/904926.

7. Ma TT, Yu SY, Li Y, Liang FR, Tian XP, et al. Randomised clinical trial: an assessment of acupuncture on specific meridian or specific acupoint vs. sham acupuncture for treating functional dyspepsia. Aliment Pharmacol Ther. 2012:35:552-61.

8. Davis RT, Churchill DL, Badger GJ, Dunn J, Langevin HM. A new method for quantifying the needling component of acupuncture treatments. Acupunct Med. 2012;30:113-9.

9. Li XM, Li YQ, Chen JX, Zhou D, Liu YY, et al. The influence of skin microcirculation blood perfusion at Zusanli acupoint by stimulating with lift-thrust reinforcing and reducing acupuncture manipulation methods on healthy adults. Evid Based Complement Alternat Med. 2013. doi:10.1155/ 2013/452697.

10. Jiang $Y$, Wang $H$, Liu Z, Dong $Y$, Dong $Y$, et al. Manipulation of and sustained effects on the human brain induced by different modalities of acupuncture: an fMRI study. PLoS One. 2013;8:e66815.

11. Hong SH, Ding SS, Wu F, Xi Q, Li Q, et al. Strong manual acupuncture manipulation could better inhibit spike frequency of the dorsal horn neurons in rats with acute visceral nociception. Evid Based Complement Alternat Med. 2015. doi:10.1155/2015/675437.

12. WHO Regional Office for the Western Pacific. WHO standard acupuncture point locations in the Western Pacific region. Manila: World Health Organization; 2008

13. Ma T, Zeng F, Li Y, Wang CM, Tian $X$, et al. Which subtype of functional dyspepsia patients responses better to acupuncture? A retrospective analysis of a randomized controlled trial. Forsch Komplementmed. 2015;22:94-100.

14. Tian XP, Li Y, Liang FR, Sun GJ, Yan J, et al. Translation and validation of the Nepean Dyspepsia Index for functional dyspepsia in China. World J Gastroenterol. 2009;15:3173-7.

15. Li L, Wang HM, Shen Y. Chinese SF-36 Health Survey: translation, cultural adaptation, validation, and normalisation. J Epidemiol Community Health. 2003;57:259-63.

16. Biggs JT, Wylie LT, Ziegler VE. Validity of the Zung Self-Rating Depression Scale. Br J Psychiatry. 1978;132:381-5.

17. Li H, Jin D, Qiao F, Chen J, Gong J. Relationship between the Self-Rating Anxiety Scale score and the success rate of 64-slice computed tomography coronary angiography. Int J Psychiatry Med. 2016;51:47-55.

18. Li XJ, Long XN, Liu LM, Chen LL, Wu Q, et al. Effect of acupuncture with different intensity on functional dyspepsia and gastric emptying. Clin J Tradit Chinese Med. 2014;9:896-9.

19. Ji X, Wang B, Lu QM. Effect of Shuganjieyu capsule combined with omeprazole on the quality of life in patients with functional dyspepsia. Chin Arch Tradit Chin Med. 2015;6:1498-500

20. Fang YJ, Liou JM, Chen CC, Lee JY, Hsu YC, et al. Distinct aetiopathogenesis in subgroups of functional dyspepsia according to the Rome III criteria. Gut. 2015:64:1517-28.

21. Shin CM. Overlap between postprandial distress and epigastric pain syndromes in functional dyspepsia: its implications for research and clinical practice. J Neurogastroenterol Motil. 2013;19:409-11.

22. Tack J, Talley NJ. Functional dyspepsia—symptoms, definitions and validity of the Rome III criteria. Nat Rev Gastroenterol Hepatol. 2013;10:134-41.

23. Han JS, Ho YS. Global trends and performances of acupuncture research. Neurosci Biobehav Rev. 2011;35:680-7.

24. Zhao ZQ. Neural mechanism underlying acupuncture analgesia. Prog Neurobiol. 2008;85:355-75

25. Xu SB, Huang B, Zhang CY, Du P, Yuan Q, et al. Effectiveness of strengthened stimulation during acupuncture for the treatment of Bell palsy: a randomized controlled trial. CMAJ. 2013;185:473-9.

26. Zaslawski CJ, Cobbin D, Lidums E, Petocz P. The impact of site specificity and needle manipulation on changes to pain pressure threshold following manual acupuncture: a controlled study. Complement Ther Med. 2003;11:11-21.
27. Kim GH, Yeom M, Yin CS, Lee H, Shim I, et al. Acupuncture manipulation enhances anti-nociceptive effect on formalin-induced pain in rats. Neurol Res. 2010;32 Suppl 1:92-5.

28. Xing WT, Wang RL. Effect of lifting-thrusting and twisting-twirling acupuncture manipulation on electrogastrogram of human. Chinese J Inf Tradit Chinese Med. 1998;2:41-2.

29. Xing WT, Mang RL. The study of effect of different manual acupuncture manipulation on electrogastrogram of human. Shanxi J Tradit Chin Med. 1991;4:33-5.

\section{Submit your next manuscript to BioMed Central and we will help you at every step:}

- We accept pre-submission inquiries

- Our selector tool helps you to find the most relevant journal

- We provide round the clock customer support

- Convenient online submission

- Thorough peer review

- Inclusion in PubMed and all major indexing services

- Maximum visibility for your research

Submit your manuscript at www.biomedcentral.com/submit 